\title{
TREATMENT OF PATHOLOGICAL SYNOVIAL PLICAE OF THE KNEE
}

\author{
Gilberto Luís Camanho
}

doi: 10.1590/S1807-59322010000300002

Camanho GL. Treatment of pathological synovial plicae of the knee. Clinics. 2010;65(3):247-50.

OBJECTIVE: To analyze the incidence, clinical significance, and clinical manifestations of pathological synovial plicae of the knee. MATERIAL AND METHODS: Between 2002 and 2006, 63 patients with pathological synovial plicae of the knee were studied. Of those 63 patients, 21 had the diagnosis confirmed by previously performed magnetic resonance imaging. All of the patients initially underwent conservative treatment for 90 days that involved strengthening and improving the flexibility of the muscles surrounding the knee as well as modification of their sports activities.

RESULTS: A total of 55 patients improved after conservative treatment. Six of these patients eventually experienced a recurrence of symptoms, but the symptoms were not incapacitating in any of these patients. The other eight patients underwent arthroscopic removal of the synovial plica. Of these eight patients, six returned to their pretreatment physical activities, and two had persistent symptoms during physical activity.

CONCLUSION: The presence of a synovial plica of the knee should be considered as a potential diagnosis in patients with knee pain, especially those who practice sports inappropriately. Conservative treatment is effective in most cases, and surgical treatment should be reserved for exceptional cases that do not improve with conservative treatment.

KEYWORDS: Synovial membrane; Synovial fluid; Knee joint; Arthroscopy; Synovitis.

\section{INTRODUCTION}

The synovial plica of the knee is formed during the embryogenic phase of development and begins to involute during the $12^{\text {th }}$ week of fetal life. This involution is incomplete in many individuals, and it has been estimated that $50 \%$ of the population has persistent plicae after birth, which can vary in shape and size. ${ }^{1,2}$ When the synovial plica of the knee persists, it is transformed into an embryonic relic that is located in either the supra- or mid-patellar region of the knee (Figure 1).

There is a syndrome that is caused by the irritation and resultant inflammatory reaction of the relic of the synovial plica of the knee that is known as "pathological synovial plica syndrome". It is characterized by supra- and mid-

Orthopedics Department, Hospital das Clinicas da Faculdade de Medicina da Universidade de São Paulo - São Paulo/SP, Brazil

Email: gcamanho@hcnet.usp.br

Tel: 55113069.6718

Received for publication on August 24, 2009

Accepted for publication on October 20, 2009

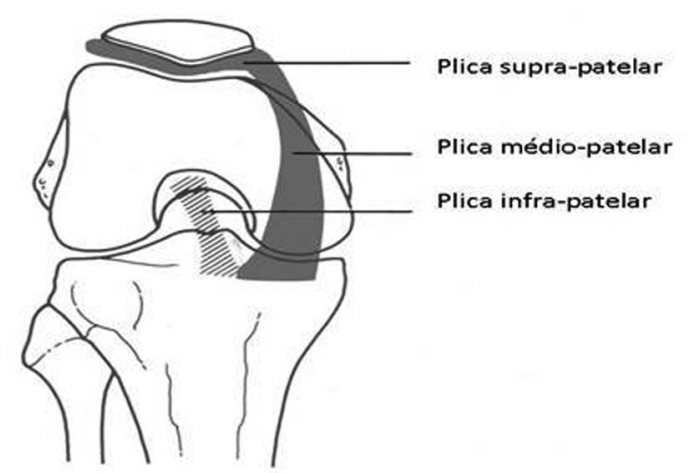

Figure 1 - Diagram of the three types of synovial plicae

patellar pain with knee extension as well as the presence of audible cracking noises with knee flexion and extension. Contraction of the quadriceps associated with compression of the supra-patellar pouch also causes pain. In addition, patients often experience a sensation of instability when they are walking up or down stairs or slopes. These symptoms have been correlated with recent changes in the intensity or quality of patients' athletic activities. 
This is a fairly frequent clinical condition among patients who perform a physical activity that is inappropriate based on their normal activities and baseline fitness level. In the past, pathological synovial plica syndrome was not treated in a uniform manner, and there was some exaggeration regarding its incidence and the need for surgical treatment. Subsequently, it became a condition that was largely forgotten among general orthopedists and one that was scarcely remembered by knee specialists. Nonetheless, it causes discomfort for an appreciable number of individuals who participate in athletic endeavors, especially those who participate in sports only occasionally.

The aim of this study was to report on our experience with 63 patients who were diagnosed with pathological synovial plica syndrome based on their clinical symptoms, of whom, 21 had the diagnosis confirmed by magnetic resonance images that had been previously performed.

\section{MATERIALS AND METHODS}

Between 2002 and 2006, we saw approximately 3200 patients with knee complaints of various etiologies in our clinic. Of these, 63 patients presented with symptoms that suggested the presence of pathological synovial plica syndrome, which was confirmed by previously performed magnetic resonance imaging in 21 of these patients. Among these 63 patients, 28 were male, 46 had symptoms in the right knee, and none had bilateral symptoms. The patients' mean age was 25.7 years (standard deviation: 5.15 years, range: $19-34$ years). The most frequent complaint was suprapatellar pain and cracking noises following the performance of physical activity of a type or intensity of which the patient was unaccustomed. Upon physical examination, all of the patients confirmed the presence of pain upon compression of the supra-patellar pouch followed by contraction of the quadriceps. A total of 14 patients reported the presence of a joint effusion following physical activity. Six patients had previously undergone arthroscopy to treat suspected meniscal lesions, and 21 patients had previously received magnetic resonance imaging examinations, none of which had been requested by the present author. At the time of the consultation, the author was able to identify synovial plicae in all of the magnetic resonance imaging scans of the 21 patients who had been previously imaged (Figure 2).

The initial treatment for all of these patients was conservative and involved the following three components: 1) ensuring that the patients' levels and types of physical activity were appropriate; 2) implementing a stretching program for the muscles of the lower extremities; and 3) providing guidance for knee extension exercises, especially terminal knee extension exercises, with the aim
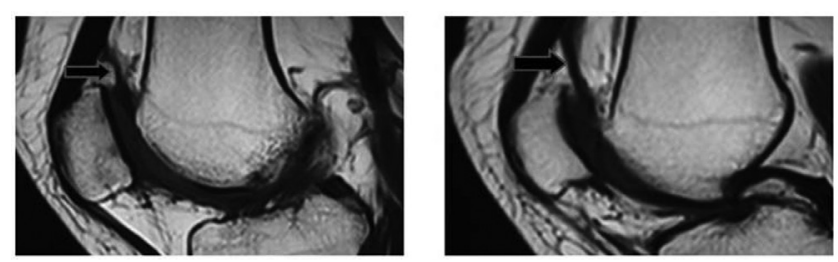

Figure 2 - Magnetic resonance image of a patient's knee with an arrow pointing to the supra-patellar synovial plica

of strengthening the tensor musculature of the joint capsule. All patients underwent a trial of conservative treatment that lasted for at least 90 days. After this period, patients were advised that they could return to their normal physical activities that they had been performing prior to the onset of their symptoms. The criterion for release from the treatment program was a patient's ability to demonstrate correct stretching of their knee flexor and extensor muscles.

Eight patients eventually underwent knee surgery because conservative treatment had not led to a resolution of their symptoms. Of these, two were from the group of patients that had already undergone arthroscopy and had not experienced any sustained improvement after three trials of conservative treatment. The surgical treatment that was employed involved the arthroscopic removal of the synovial plica and a postoperative rehabilitation program that was identical to the one used in the conservative treatment regimen. This program was started, on average, 15 days after the surgical procedure was performed (Figures 3 and 4).

All of the patients were followed up with by the present author for at least two years after the onset of their symptoms.
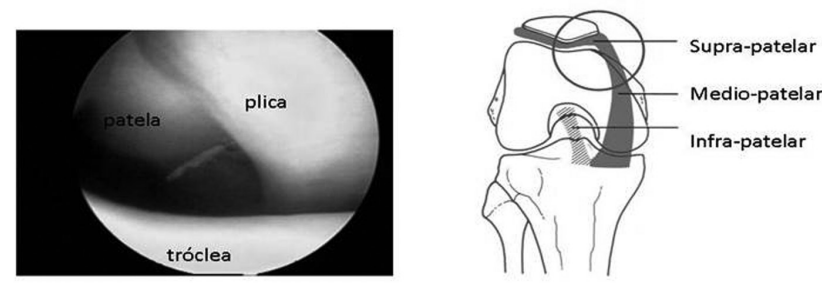

Figure 3 - Arthroscopic image of a mid-patellar synovial plica. The patella can be seen above the femoral trochlea, with a large mid-patellar plica interposed. In the drawing, the circle demonstrates the position of the arthroscopies
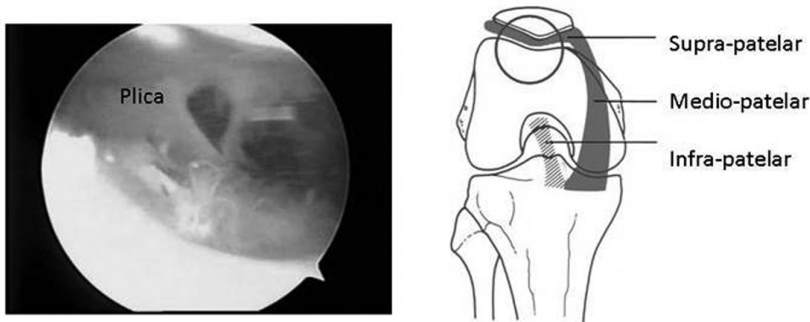

Figure 4 - Arthroscopic image of a supra-patellar synovial plica. The patella can be seen above the femoral trochlea. In the drawing, the circle demonstrates the position of the arthroscopy 


\section{RESULTS}

\section{Patients treated exclusively by conservative means:}

55 patients:

- 49 returned to their previous physical activities without complaints of symptom recurrence;

- 6 returned to their previous physical activities with sporadic complaints of symptom recurrence that was not incapacitating.

\section{Patients who underwent surgical management:}

8 patients:

- 6 returned to their previous physical activities without complaints of symptom recurrence;

- 2 were unable to return to their previous physical activities.

\section{DISCUSSION}

The synovial plica of the knee was the first described by Iino $^{3}$ in 1939, who also classified it into four different subtypes. For this reason, some authors have referred to the synovial plica of the knee as "Iino's band". The prevalence of synovial plicae of the knee, which are embryonic relics, has been studied by several authors, who have all found them to be present in about $50 \%$ of the population. ${ }^{2}$ Interestingly, several Asian studies have observed the presence of synovial plicae in higher percentages of the population. For example, Kim and $\mathrm{Choe}^{4}$ described an incidence of synovial plicae of $87 \%$ in their cohort in a study using arthroscopy. Jackson et al. ${ }^{5}$, in a joint Canadian and Japanese study, found 206 cases of synovial plica $(60 \%)$ in 345 patients undergoing arthroscopic surgery. They reported that there were no racerelated differences in the prevalence of synovial plicae.

Gurbuz et al. ${ }^{6}$ reported an unequal distribution of the type of plica (i.e., supra-, mid-, or intra-patellar, with the designation based on the embryonic relic that remained) that was present among their patients. They also reported seven bilateral cases. Their study was performed on cadavers and utilized arthroscopy to visualize the plicae. Within our setting, Cardoso ${ }^{2}$ found that $48 \%$ of the cadavers they examined had supra- or mid-patellar plicae. In another study that included 523 patients undergoing arthroscopic procedures, Cohen et al. ${ }^{7}$ found that $18 \%$ of patients (94) had plicae. Supra- and mid-patellar plicae are of more clinical interest to physicians because they are the types of plicae that can become symptomatic.

Patients with pathological synovial plica syndrome have a very characteristic clinical presentation, and their symptoms are always related to physical activity. ${ }^{8}$ In addition to the symptoms described earlier in the paper, Pekmezci et al. ${ }^{9}$ and Adachi et al. ${ }^{10}$ have described additional symptoms, including significant lateral knee pain and a sensation of joint "locking".

The diagnosis of this condition is essentially a clinical one that is based on patients' history and symptoms. This syndrome is caused by excessive or inappropriate use of the $\mathrm{knee}^{9}$. If a plica is unexpectedly visualized during arthroscopy for an unrelated reason, it should not be removed because about $50 \%$ of the population has plicae, and plicae are almost always asymptomatic. Denti et al. ${ }^{11}$ compared the clinical outcomes of plicae resection in patients with pathological synovial plicae syndrome with those of resection of plicae in patients who underwent arthroscopy for unrelated reasons and were found to have a plica. The former group of patients had good clinical outcomes $90 \%$ of the time, whereas only $30 \%$ of patients in the latter group did.

The magnetic resonance imaging scans of the 21 patients who had previously undergone the test at the request of another physician were analyzed during the initial consultation. The presence of a synovial plica was clear in all of the patients' scans. Although magnetic resonance imaging is an efficient way to detect synovial plicae, the mere observation of the presence of synovial plicae in the absence of the characteristic clinical symptoms should never be considered to be an indication for surgery.

Most patients were managed successfully with conservative treatment. The conservative management of these patients has been previously recommended by Amatuzzi et al. ${ }^{12}$ Arthroscopic resection should be reserved for exceptional cases, and, when it is necessary, it generally has good outcomes. ${ }^{13}$ Only eight of our patients failed conservative management and required surgery, and six of them obtained full relief of their symptoms postoperatively.

Our cohort included six patients who had previously undergone surgical treatment to correct meniscal lesions. Of these, four experienced good results from their trial of conservative treatment, one had a good result following surgical treatment, and the remaining patient did not have a good outcome, as this patient experienced persistent symptoms during physical activity.

Some authors ${ }^{13,14}$ have reported on case studies in which a large proportion of patients were treated surgically. However, the consensus in the literature is that conservative treatment should be attempted before surgery is recommended.

\section{CONCLUSION}

Pathological synovial plica syndrome should be considered to be caused by excessive use of the knee. Surgical treatment should only be considered in exceptional cases that do not respond to conservative treatment. 


\section{REFERENCES}

1. Camargo OPA. Pregas sinoviais hipertróficas. In: Camanho GL. Patologia do joelho. São Paulo: Sarvier; 1996. p.74-7.

2. Cardoso TP, Camanho GL. Incidência da prega sinovial medial do joelho: estudos em cadáveres. RBO, 1996;31:169-74.

3. Iino S. Normal arthroscopic findings in the knee joint in adult cadavers. J Japanese Orthop Assn. 1939;14:467-523.

4. Kim SJ, Choe WS. Arthroscopic findings of the synovial plicae of the knee..Arthroscopy. 1997;13:33-41.

5. Jackson RW, Marshall DJ, Fujisawa Y. The pathologic medial shelf. Orthop Clin Nort Am. 1982;13:307-12.

6. Gurbuz H, Calpur OU, Ozcan M, Kutoglu T, Mesut R. The synovial plicae in the knee joint. Saudi Med J. 2006;27:1839-42.

7. Cohen M, Abdalla RJ, Queiroz AAB, Saone R. Estudo artroscópico da prega synovial sintomática do joelho.Rev Bras Ortop. 1987;22:293-6.

8. Bae DK, Nam GU, Sun SD, Kim YH. The clinical significance of the complete type of suprapatellar membrane. Arthroscopy. 1998;14:830-5.
9. Pekmezci M, Atay OA, Kerimoğlu U, Aydingöz U, Tetik O, Doral MN. A complete supra-patellar plica with an unusual presentation. Knee Surg Sports Traumatol Arthrosc. 2006;14:872-4.

10. Adachi N, Ochi M, Uchio Y, Kawasaki K, Yamasaki K. The complete type of suprapatellar plica in a professional baseball pitcher: consideration of a cause of anterior knee pain. Arthroscopy. 2004;20:987-91.

11. Denti M, Monteleone M, Berardi A, Arosio A. Medial patellar synovial plica syndrome: the influence of associated pathology on long-term results. Chir Organi Mov. 1994;79:273-7.

12. Amatuzzi MM, Fazzi A, Varella MH. Pathologic synovial plica of the knee. Results of conservative treatment. Am J Sports Med. 1990;18:4669.

13. Hansen H, Boe S. The pathological plica in the knee. Results after arthroscopic resection. Arch Orthop Trauma Surg. 1989;108:282-4.

14. Amatuzzi MM, Albuquerque RF. [Pathologic synovial plica of the knee. Results of surgical treatment in 37 knees]. Rev Hosp Clin Fac Med São Paulo. 1994;49:104-6. 